ORIGINAL ARTICLE

\title{
Twenty years of a multidisciplinary paediatric diabetes home care unit
}

\section{A McEvilly, J Kirk}

See end of article for authors' affiliations

Correspondence to: Dr J Kirk, Department of Diabetes, Birmingham Children's Hospital, Steelhouse Lane, Birmingham $\mathrm{B} 4$ ' $6 \mathrm{NH}$, UK Jeremy.Kirk@bch.nhs.uk

Accepted 29 September 2004

As only a minority of patients with type 1 diabetes are unwell at diagnosis, these patients could be managed at home if appropriate facilities were available. A multidisciplinary diabetes home care service was established over 20 years ago at Birmingham Children's Hospital, to support children with diabetes mellitus within the home environment from diagnosis, reducing emotional upset and separation. Despite increase in the size and distribution of the unit over this time (from 230 to 400 patients (now spread over two hospitals)), the proportion of newly diagnosed children managed wholly at home (median 43\%; range $31-67 \%$ ), and the reduction in number and duration of admissions has been sustained (readmission rate with diabetic ketoacidosis 4.1 bed-days per 100 patients/year; range 2.9-7.1), with no deterioration in overall blood glucose control. In this way the savings achieved by reductions in expensive hospital bed occupancy have more than offset the costs of maintaining the unit.

A lthough outpatient management of insulin dependent diabetes mellitus has been advocated for many decades, ${ }^{1}$ most children still have a period of inpatient stabilisation at diagnosis, regardless of their clinical condition. ${ }^{2}$ Recently the shift in emphasis away from hospital to home management of children with chronic and non-urgent conditions has recognised the need for hospital based, community orientated paediatric services. ${ }^{3}$

As fewer than $30 \%$ of children with newly diagnosed diabetes require hospital admission for medical reasons, ${ }^{4}$ if insulin therapy could be commenced at home, then in the majority of cases it would be unnecessary to admit an otherwise well child. Disruption to the child and family could therefore be kept to a minimum.

In 1981, in response to this perceived need, a diabetes home care (DHC) service was established at the Children's Hospital in Birmingham. The benefits of the unit were initially described by Rayner in 1984, ${ }^{5}$ who showed, in addition to an improvement in metabolic control, that the potential savings in reduction in bed-days for new and established patients with diabetes outweighed the costs of the service itself.

Since 1984 there has not only been a substantial increase in the number of patients and staff in the unit, but the service has now been successfully extended to another unit within the city. ${ }^{6}$ We therefore wished to see whether the initial improvements described in 1984 have been sustained over a period of greater than 20 years.

FUNCTIONS OF THE DIABETES HOME CARE SERVICE At its inception the DHC unit at the Children's Hospital cared for 230 children with diabetes. In 1995 the service was both extended across Birmingham to another unit (City Hospital, 40 patients), and also absorbed the 40 paediatric patients from another unit (Selly Oak Hospital). The service now cares for almost 400 children with diabetes (350 at the Children's Hospital and 50 at City Hospital); table 1 shows the previous and present structure. The workload and activity of the unit has also risen during this time (see table 2).

Members of the DHC team are available 24 hours a day, either through their direct departmental line when in the hospital, or via the hospital paging service. The paediatric diabetes specialist nurses (PDSNs) work in a flexible shift system four days a week (07 30-17 30 and 10 15-20 00), with one on-call at the weekend, and are the first line of contact. On-call duties are paid at a nominal rate. The consultants provide a medical on-call l-in-3 for endocrinology and diabetes, and also cover the PDSNs at times of holiday, illness, or study leave.

\section{Newly diagnosed patients}

On diagnosis children are initially reviewed and assessed by the DHC team. If admission is not medically required, wherever possible they return home with the PDSN to commence treatment and education in familiar surroundings. It is not usual for medical staff to attend these home visits.

Admission to hospital is necessary for those children who develop diabetic ketoacidosis or are otherwise unwell. Other occasions when a period of inpatient management might be more appropriate include adverse social circumstances and communication difficulties (as experienced with some asylum seekers). In addition, there may be logistic problems such as staff shortages (illness, study leave, holiday, etc), or several new patients presenting simultaneously, and in these situations a decision would be made by the team based on the individual circumstances.

For those patients who require admission, early contact on the ward is made by the PDSN, and the child discharged when medically fit under their care (usually within 2448 hours).

Any home support of the newly diagnosed child is provided at injection times for the first few days, to assist the family as

Table 1 Structure of DHC unit

\begin{tabular}{lll}
\hline Year & $\mathbf{1 9 8 4}$ & $\mathbf{2 0 0 3}$ \\
\hline No. of patients & 230 & 400 \\
Sites & 1 & 2 \\
Staff (WTE) & 1.0 & 2.6 \\
Senior medical staff & 2.0 & 4.0 \\
PDSN & 0.5 & 1.5 \\
Dieticians & 1.0 & 1.6 \\
Administrative &
\end{tabular}




\begin{tabular}{|c|c|c|c|c|c|c|c|c|c|c|c|c|}
\hline Year & 1981 & 1983 & 1992 & 1995 & 1996 & 1997 & 1998 & 1999 & 2000 & 2001 & 2002 & 2003 \\
\hline $\begin{array}{l}\text { Total patient numbers } \\
\text { (BCH only) }\end{array}$ & 230 & 220 & & & & & 250 & 263 & 274 & 310 & 340 & 350 \\
\hline \multicolumn{13}{|l|}{ Home visits } \\
\hline Emergency & & & & 194 & 160 & 171 & 170 & 129 & 195 & 153 & 166 & 181 \\
\hline Planned & & & & 555 & 585 & 566 & 569 & 523 & 423 & 462 & 497 & 567 \\
\hline Total & & & 585 & 747 & 745 & 737 & 739 & 652 & 618 & 615 & 663 & 748 \\
\hline School visits & & & & 61 & 57 & 64 & 81 & 71 & 72 & 90 & 90 & 94 \\
\hline Ward visits & & & 172 & & 221 & 250 & 175 & 216 & 177 & 186 & 138 & 206 \\
\hline Outpatient visits & & & & 808 & 800 & 895 & 987 & 942 & 951 & 984 & 1048 & 986 \\
\hline Department visits & & & & 109 & 89 & 98 & 187 & 226 & 300 & 388 & 305 & 321 \\
\hline Drop-in & & & & & & & & & & 145 & 195 & 156 \\
\hline
\end{tabular}

they develop their practical skills and to provide reinforcement of advice and education. Care and education is adapted to the individual child and family, making it relevant to their specific needs. Home support at injection times usually continues for 2-3 days and is then followed by a visit at a non-injection time to continue education at a less stressful time. Dietetic support is provided in the home within the first 4-5 days, and follow up visits are undertaken jointly with the PDSN when appropriate. All families are made aware of how to contact the DHC nurses and are encouraged to do so if concerned, as well as being provided with guidelines about when to get advice. Six weeks following diagnosis the family's knowledge is formally assessed using a validated multiple choice questionnaire, and education reviewed as necessary.

\section{Existing patients}

Continuing support of patients is provided by twice yearly home visits. These have been achieved in over $70 \%$ of patients; the remainder have all been visited once. Regular telephone contact ( $>7000$ calls/year) is also kept, although there is considerable variation in frequency of contact with individual patients.

In addition to home visits, routine monitoring and assessment of diabetes control is also undertaken at the outpatient clinic, where the family meets the entire DHC team (PDSN, doctor, dietician). Clinics are age banded to provide transitional care, with adolescent clinics held in the afternoon and evening. Following planning for transfer, support is also provided in the young adult clinics as the young people move to adult care.

An informal nurse-led drop in clinic has now been established on Saturday morning at a time when the nurses are already hospital based, where the families can call in to discuss any aspect of diabetes and its management. Interestingly the adolescent patient group have mainly accessed this service (see table 2 ).

While the service was established to provide care within a 10 mile radius of the Children's Hospital (an area approximating to the City of Birmingham), due to demand there have been a number of children referred to the service from outside this area. The majority of these are established patients with diabetes (tertiary referrals) and who are experiencing problems. While the team are unable to offer home support to these children, they are reviewed twice yearly within the department, at outpatient clinics, and also maintain telephone contact.

\section{OTHER FUNCTIONS OF THE DHC SERVICE}

The team has developed protocols to ensure continuity of care within the team and the hospital. The PDSNs work within these protocols (as agreed by the hospital clinical governance department). For those children experiencing poor glycaemic control, the nurses follow additional guidelines for support.

Like other units, ${ }^{7}$ the clinic at the Children's Hospital is seeing an increase, not only in the number of children with type 1 diabetes, but also in those with more unusual forms (type 2 and maturity onset diabetes of the young (MODY)), and the home care team is responsible for these children, as well as those who develop diabetes in the course of, or during treatment for other diseases.

Ongoing support and education are provided to other healthcare professionals, in both the hospital and community (including schools and general practitioners). For the past 14 years the team has been running the Management of Childhood Diabetes Course for the training of nurses and dieticians involved in the care of children with diabetes, and in 2002 the team also established an advanced course for the training of specialist registrars and newly appointed consultants.

\section{RESULTS (SEE FIG 1 AND TABLE 2) \\ Bed-days}

Rayner reported the early results and benefits of this service in 1984, ${ }^{5}$ at which time all new diabetic patients were admitted for stabilisation. With the development of the service, total inpatient bed-days fell from 555 to 127 per year. There was also a reduction in the length of stay for a newly diagnosed child, from a mean of 12.2 days in 1980 to 4.1 days in 1984.

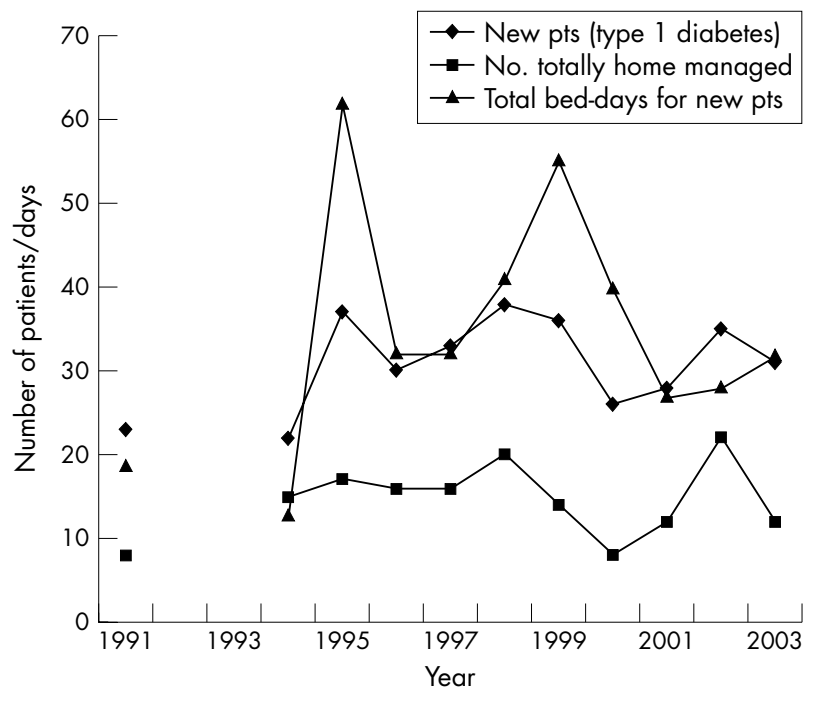

Figure 1 New patients: number of patients, number totally home managed, and bed-days. 
By 1994 the average was 0.6 bed-days at diagnosis for new patients, with $66.6 \%$ of the patients being totally home managed at diagnosis (compared with $33.3 \%$ in 1989). This reduction in bed-days has been sustained: in 2002 there were 28 bed-days for 35 new diabetic patients ( 0.8 bed-days per patient). In 200312 of 31 newly diagnosed patients (39\%) were totally home managed. Readmission rates have also fallen, from a initial mean of 7.2 days per patient in 1980 to 2.3 days three years later. In 1994 the readmission rate was 0.2 bed-days per patient, as it was in 2002. For readmission with diabetic ketoacidosis the median number of total beddays for all patients over the last three years has been 14/year (range 10-24), a readmission rate of 4.1 bed-days per 100 patients (range 2.9-7.1).

When the admission rates for newly diagnosed children have increased, these are often associated with times of staff shortages, high incidence of new patients (particularly the under 5 year olds), and an increase in the number of newly diagnosed children with ketoacidosis; ${ }^{8}$ approximately $50 \%$ of our new patients require admission for medical reasons. In addition, as bed-days are assessed as bed occupancy at midnight, this undoubtedly also skews the "totally" home managed percentage figures downwards, as many patients presenting after $5 \mathrm{pm}$ have been admitted briefly overnight, and home managed from the next morning. Increased readmission rates often relate to a small number of specific patients experiencing particular problems (including increasing numbers of tertiary referrals of poorly controlled patients).

In terms of nursing time, there was no significant difference whether children were admitted at diagnosis or not. In 1994 in the first two weeks following diagnosis, those children admitted to hospital for stabilisation received an average of 11.6 hours of intensive nursing contact, compared with 12.1 hours (excluding travel time) for those supported at home from diagnosis.

\section{Diabetic control}

Glycosylated haemoglobin (HbAl from 1981 to 1994, HbAlc from 1994 onwards) is routinely measured 3-6 monthly, and more frequently in poorly controlled patients. The initial results published in 1984 showed a reduction in mean $\mathrm{HbAl}$ from $12.8 \%$ to $11.6 \%$. Overall the $\mathrm{HbAl}$ remained lower, at $10.4 \%$ in 1987 and $10.5 \%$ in 1993. Between 1994 and 1997 glycosylated haemoglobin was measured using an accredited laboratory based method. Since 1997 an equivalent assay, using the DCA-2000 meter (Bayer AG, Germany) was used to provide bedside testing (again measured every 3-6 months). From 2000 onwards the mean HbAlc has been between 9.0\% and $9.3 \%$, although this increase may well reflect a change in the protocol for measuring HbAlc.

\section{Cost effectiveness}

The original paper by Rayner described a potential saving in 1984 of $£ 26415$, based on the estimated saving of in-patient expenditure ( $£ 52917$ ), less the cost of the service itself $(£ 26502) .^{5}$

In 1995 the 40 patients from a unit in another district (Selly Oak Hospital) were absorbed into the DHC unit with no additional resources. Prior to the merger this district had an average of 10.4 bed-days for newly diagnosed patients (compared to 1.7 for $\mathrm{BCH}$ ). The average readmission beddays the year prior to transfer were 2.7, compared to 0.2 for the DHC. On this basis the savings per diabetic patient (at $£ 419$ per bed-day) were estimated to be $£ 1129.50$ per year (even allowing for the increased nursing input). These figures were extrapolated from potential savings as hospital beds not occupied by children with diabetes were freed up for other hospital admissions, thereby releasing pressure on departments both in our hospital and elsewhere. In the year after merger none of the Selly Oak patients were readmitted.

These savings have been confirmed using the figures from 2002. Even allowing for the trend towards reduction in hospital stay of newly diagnosed diabetics, the most recent data from the Audit Commission (2000) showed a mean stay of 3.0 bed-days/patient ${ }^{9}$ (based on seven units), compared to 0.8 for the home care unit. For existing patients (based on our own data and that from Selly Oak) the readmission rate for existing patients is 2.0 bed-days/patient/year (compared to 0.2 for the DHC service). The savings are therefore 2.2 beddays for 35 newly diagnosed patients ( 77 bed-days) and 1.8 bed-days for 350 existing patients (628 bed-days); a total of 705 bed-days. At a current cost of $£ 500$ per diabetic bed-day this represents a potential saving of $£ 355500$, which outweighs the total cost of running the unit.

\section{DISCUSSION}

The concept of specialised teams to provide care for children with diabetes was proposed in 1989 at St Vincent in Italy, ${ }^{10}$ and was subsequently endorsed in a number of national and international guidelines. ${ }^{11-13}$ In 2000 the Audit Commission not only advocated home management whenever possible, but also flagged up the home care service at $\mathrm{BCH}$ as a model in its case studies. ${ }^{9}$

Paediatricians have long recognised the differences between adult and child patients, and the need to address these differences by managing the paediatric population with dedicated paediatric teams..$^{14}$ As a further development in this attitude, there has been a pronounced shift away from favouring entirely hospital based care for children with chronic conditions, now highlighted in the Standards for the National Service Framework (NSF) for Children. ${ }^{14}$

At the time of the establishment of the home care service in Birmingham, total home management for the newly diagnosed child was not considered, as it was felt patients and parents would benefit from a period of time in hospital to come to terms with the diagnosis of diabetes. Since that time, however, the team has cared for between $31 \%$ to $67 \%$ of newly diagnosed children at home, despite increasing numbers.

The philosophy of home care benefits the newly diagnosed in limiting separation and allows the family to be "in control" from the outset, enabling them to absorb knowledge more easily in the home environment. Established patients have the confidence to contact the team with whom they have built up a relationship since diagnosis, leading to more home management at times of difficulty. Whether the reduced readmission rate reflects knowledge acquired better in a home environment, and/or easy access to 24 hour advice is unclear, but this has been very apparent when the service has taken over the care of children from other areas, where time is required to enable the child and family to build confidence with the team, enabling them to manage problems at home rather than bringing the child to hospital. In addition the rationale of care at home enables the whole family to be involved, and enables the nurses and dieticians to see how the family live and how diabetes care will be managed by their lifestyle.

In our unit, with a large paediatric diabetic population and commensurate staff size, it is possible to provide a truly home based service for many of these patients. An alternative (especially in smaller units) might be for patients to remain at home but to return to the unit for injections and education. This will reduce the need for hospital beds for newly diagnosed patients, although whether readmission of existing patients will also be reduced is unclear. Our service does not currently provide this service. 
There has already been published evidence of the benefit of short term versus long term admissions, ${ }^{15}$ especially where specialist nurses in diabetes are available, ${ }^{16}$ and of home versus hospital based care of the newly diagnosed child with diabetes. ${ }^{17}$ No advantage has been shown in favour of hospital admission in terms of parental satisfaction, glycaemic control, or long term outcomes. We have already shown by questionnaire (data not shown) that children and families prefer home centred management. Indeed, there is increasing evidence that outcomes are in fact improved in patients who have initial management outside hospital, ${ }^{18}$ and we have now shown clear financial benefits of such arrangements. Consequently, the National Institute for Clinical Excellence (NICE), in its draft guidelines for type 1 diabetes (www. nice.org.uk) has now stated: "At the time of diagnosis children and young people with type 1 diabetes should be offered home-based or inpatient management according to clinical need, family circumstances and wishes, and residential proximity to inpatient services. Home-based care with support from the local paediatric diabetes care team (including 24-hour telephone access to advice) is safe and as effective as inpatient initial management" ${ }^{\prime 1}{ }^{19}$

The overall control (assessed using HbAlc) in patients who have been totally home managed compares favourably with other units. Over the last three years, however, the mean clinic HbAlc has worsened significantly within the unit: this may, however, reflect the new unit protocol for more frequent measurement of HbAlc in poorly controlled patients. In the National Paediatric Diabetes Audit in 2001 the mean case mix adjusted residual for $\mathrm{HbAlc}$ for our centre was the 12th best of 97 units in the UK, and also compares favourably with large international units. ${ }^{20}$

For the diabetes home care service the aim is to sustain and improve the quality of care provided for the children with diabetes in Birmingham. This will involve both tried and proven training, but also embracing of new technologies, ${ }^{21}$ to ensure that outcomes are brought into line with the recommendations the Diabetes Control and Complications Trial. $^{22}$

In conclusion, we have shown that a multidisciplinary paediatric diabetes team enables patients, whenever possible, to be managed at home from diagnosis, reducing hospital admission not only for new, but also for existing patients. This results in significant potential savings in bed-days for new and existing patients. In addition, the overall control of these patients compares very favourably with other equivalent units.

\section{ACKNOWLEDGEMENTS}

We would like to thank all the staff, patients, and parents who have contributed to the success of the unit since its inception in 1981 .

\section{Authors' affiliations}

J M W Kirk, A E McEvilly, Department of Diabetes/Endocrinology,

Diana, Princess of Wales Children's Hospital, Birmingham, UK

Competing interests: none declared

\section{REFERENCES}

1 Walker JB. Field work of a diabetic clinic. Lancet 1953;ii:445-7.

2 Clar C, Waugh N, Thomas S. Routine hospital admission versus out-patient or home care for children diagnosis of type 1 diabetes mellitus. Cochrane Database Syst Rev 2003;(3):CD004099.

3 Meates M. Ambulatory paediatrics-making a difference. Arch Dis Child 1997;76:468-73.

4 Smith CP, Firth D, Bennett $S$, et al. Ketoacidosis occurring in newly diagnosed and established diabetic children. Acta Paediatr 1998;87:537-41.

5 Rayner PHW. A home care unit for diabetic children. Practical Diabetes 1984;1:5-6.

6 Kirk J, Thomas E, McEvilly A, et al. Extension of a paediatric diabetes home care service. Practical Diabetes International 2003;20(4):125-8.

7 Gardner SG, Bingley PG, Sawtell PA, and the Bart's Oxford Study Group, et al. Rising incidence of insulin-dependent diabetes in children aged five years in the Oxford region: time trend analysis. BMJ 1997;315:713-17.

8 Alvi N, Davies P, Kirk JM, et al. Diabetic ketoacidosis in Asian children. Arch Dis Child 2001;85:60-1.

9 Audit Commission. Testing times. A review of diabetes services in England and Wales. London: Audit Commission, 2000.

10 Betts P, Buckley M, Davies R, et al. The care of young people with diabetes (St Vincent and improving diabetes care specialist UK workgroup reports). Diabet Med 1996;13(suppl 4):S54-9.

11 British Diabetic Association. The principles of good practice for the care of young people with diabetes. London: British Diabetic Association, 1995.

12 ISPAD. Consensus guidelines for the management of type 1 mellitus in children and adolescents. Zeist, Netherlands: Medical Forum International, 2000.

13 National Service Framework (NSF) in Diabetes. http://www.doh.gov.uk/ nsf/diabetes.

14 National Service Framework (NSF) in Children Standards. http:// www.doh.gov.uk/nsf/children.htm.

15 Simell T, Kaprio EA, Maenpaa J, et al. Randomised prospective study of shortterm and long-term stay in hospital by children with diabetes mellitus. Lancet $1991 ; 337: 656-60$.

16 Lipman TH. Length of hospitalisation of children with diabetes: effect of a clinical nurse specialist. Diabetes Educator 1986;14:41-3.

17 Swift PGF, Hearnshaw JR, Botha JL, et al. A decade of diabetes: keeping children out of hospital. BMJ 1993;307:96-8.

18 McNally PG, Burden AC, Swift PGF, et al. The prevalence and risk factors associated with the risk of diabetic nephropathy in juvenile-onset (insulin dependent) diabetics diagnosed under the age of 17 years in Leicestershire 1930-1985. QJM 1990;76:831-44.

19 National Institute for Clinical Excellence (NICE). CG15 type 1 diabetes: diagnosis and management of type 1 diabetes in children and young people. NICE guideline, July 2004. http://www.nice.org.uk/pdf/ Typeldiabetes(child)Fullguideline.pdf.

20 Danne T, Mortensen HB, Hougaard P, et al. Persistent differences among centres over 3 years in glycaemic control and hypoglycaemia in a study of 3,805 children and adolescents with type 1 diabetes from Hvidore Study Group. Diabetes Care $2001 ; 24: 1342-7$.

21 Scott A, Donnelly R. Improving outcomes for young people with diabetes: use of new technology and a skills-based training approach is urgently needed. Diabet Med 2001;18:861-3.

22 Diabetes Control and Complications Trial (DCCT) Research Group. The effect of intensive treatment of diabetes on the development and progression of longterm complications in insulin-dependent diabetes mellitus. N Engl J Med 1993;329:977-86 